\title{
Genome-wide identification of the GATA transcription factor family and their expression patterns under temperature and salt stress in Aspergillus oryzae
}

\author{
Chunmiao Jiang, Gongbo Lv, Jinxin Ge, Bin He, Zhe Zhang, Zhihong Hu and Bin Zeng * (]
}

\begin{abstract}
GATA transcription factors (TFs) are involved in the regulation of growth processes and various environmental stresses. Although GATA TFs involved in abiotic stress in plants and some fungi have been analyzed, information regarding GATA TFs in Aspergillus oryzae is extremely poor. In this study, we identified and functionally characterized seven GATA proteins from A. oryzae 3.042 genome, including a novel AoSnf5 GATA TF with 20-residue between the Cys- $\mathrm{X}_{2}$-Cys motifs which was found in Aspergillus GATA TFs for the first time. Phylogenetic analysis indicated that these seven A. oryzae GATA TFs could be classified into six subgroups. Analysis of conserved motifs demonstrated that AspergilI us GATA TFs with similar motif compositions clustered in one subgroup, suggesting that they might possess similar genetic functions, further confirming the accuracy of the phylogenetic relationship. Furthermore, the expression patterns of seven A. oryzae GATA TFs under temperature and salt stresses indicated that A. oryzae GATA TFs were mainly responsive to high temperature and high salt stress. The protein-protein interaction network of A. oryzae GATA TFs revealed certain potentially interacting proteins. The comprehensive analysis of A. oryzae GATA TFs will be beneficial for understanding their biological function and evolutionary features and provide an important starting point to further understand the role of GATA TFs in the regulation of distinct environmental conditions in A. oryzae.
\end{abstract}

Keywords: Aspergillus oryzae, GATA transcription factors, Temperature stress, Salt stress, Gene expression

\section{Introduction}

GATA transcription factors (TFs) constitute a family proteins that is characterized by the presence of one or two highly conserved type-IV zinc fingers $\left(\mathrm{Cys}-\mathrm{X}_{2}-\mathrm{Cys}-\mathrm{X}_{17-20^{-}}\right.$ Cys- $\mathrm{X}_{2}$-Cys) and a DNA-binding domain that recognizes the conserved (A/C/T)-G-A-T-A- (A/G) sequence in the promoter sequence of target genes (Scazzocchio 2000; Lowry and Atchley 2000). In fungi, GATA TFs are mainly involved in nitrogen regulation and light responses,

\footnotetext{
*Correspondence: zengtx001@aliyun.com

Jiangxi Key Laboratory of Bioprocess Engineering and Co-Innovation Center for In-Vitro Diagnostic Reagents and Devices of Jiangxi Province, College of Life Sciences, Jiangxi Science and Technology Normal University, Nanchang 330013, China
}

regulation of sexual and/or asexual reproduction, and secondary metabolism. GATA TFs AreB and AreA are not only involved in nitrogen and carbon metabolism, but also in the control of several complex cellular processes such as transport and secondary metabolism (SM) (Pfannmüller et al. 2017; Chudzicka-Ormaniec et al. 2019). SreA is involved in the regulation of siderophore biosynthesis and iron uptake (Oberegger et al. 2010; Schrettl et al. 2008), and $N s d D$ regulates sexual and/or asexual reproduction and the production of SMs (Lee et al. 2014, 2016; Niehaus et al. 2017). Furthermore, few fungal GATA TFs also play important roles in response to abiotic stresses. Alternaria alternata SreA is related to the maintenance of cell wall integrity (Chung et al. 2020), while Blastomyces dermatitidis SreB strongly expresses 
and contributes to filamentous growth at $22{ }^{\circ} \mathrm{C}$ via lipid metabolism (Marty et al. 2015). Additionally, GLN3 and GAT1 have been shown to be involved in salt tolerance in Saccharomyces cerevisiae (Crespo et al. 2001). However, reports regarding the function of GATA TFs filamentous fungi in response to abiotic stress factors are limited.

Aspergillus oryzae is an important filamentous fungus, which is widely used in East Asian traditional fermented food products (Kitamoto 2015). During fermentation, A. oryzae is exposed to various environmental stress factors. Temperature is the most important environmental factor affecting the growth and activity of microorganisms and can directly affect the activity of enzymes involved in substrate digestion during fermentation process (Chen et al. 2011; Bechman et al. 2012). In addition, high sodium chloride concentration, which inhibits the growth of spoilage bacteria in soy sauce mash, also affects the growth of $A$. oryzae (Wang et al. 2013; Fernandes et al. 2018). Therefore, the ability of $A$. oryzae to adapt to different temperatures and high salt concentrations have attracted attention, although the molecular mechanisms underlying their response to these stress factors still remain unclear. Previous studies have demonstrated that GATA TFs are mainly involved in regulation of various temperature and salt stress stimuli in few fungi (Scazzocchio 2000; Crespo 2001; Marty et al. 2015). Although the Fungal Transcription Factor Database (FTFD) and Kobayashi et al. have publicized six A. oryzae GATA $\mathrm{TFs}$, which are involved in nitrogen regulation, light responses, regulation of sexual and/or asexual reproduction, and SM (Kobayashi et al. 2007), studies regarding a comprehensive analysis of $A$. oryzae 3.042 GATA TFs are lacking. Therefore, the aim of this study was to analyze the structural characteristics, evolutionary features, conserved motifs, and expression patterns of A. oryzae GATA TFs under temperature and salt stress. Furthermore, the expression patterns and the results of proteinprotein interaction (PPI) can establish a good foundation for further studies on the function and the mechanism of A. oryzae GATA TFs in abiotic stress responses.

\section{Materials and methods}

\section{Identification of $A$. oryzae GATA TFs}

The A. oryzae 3.042 genome was downloaded from NCBI database (https://www.ncbi.nlm.nih.gov/genome/? term $=$ Aspergillus + oryzae). The BLASTP program, with a threshold e-value of 1e-10, was used to predict GATA TFs in the A. oryzae 3.042 genome, using gene sequences from Aspergillus as query sequences. All potential $A$. oryzae GATA TFs were identified using HMMER3.1 and were predicted if they contained ZnF-GATA domains (PF00320). The sequences that generated hits with GATA-type zinc finger genes encoding GATA zinc-finger domains (PF00320) were considered as GATA TFs. CDD and PFAM databases were used to validate all the potential $A$. oryzae GATA TFs. Finally, seven query sequence IDs of Ao3042_00752, Ao3042_04581, Ao3042_01136, Ao3042_04436, Ao3042_04150, Ao3042_05944, and Ao3042_05500 contain ZnF-GATA domains.

To determine the chromosomal locations of the seven identified A. oryzae GATA TFs, locus coordinates were downloaded from the A. oryzae RIB40 genomics database. The distribution of seven $A$. oryzae TFs on the chromosomes was drawn using MG2C (mg2c.iask.in/ mg2c_v2.0/) and visualized using MapChart 2.2 (Voorrips 2002).

\section{Multi sequence alignment and phylogenetic analysis}

ClustalW was used to align A. oryzae GATA TF proteins. The protein sequences of known GATA TFs in all other Aspergillus were downloaded from fungal transcription factor databases (FTFD, http://ftfd.snu.ac.kr/index. php? $\mathrm{a}=$ view). The sequences of GATA TFs in A. oryzae and other Aspergillus species were also aligned using ClustalW to analyze the phylogenetic relationships of all Aspergillus GATA TFs. A neighbor-joining (NJ) tree was constructed based on alignment results in MEGA6.0 with bootstrap replications of 1000 . The sequence IDs of GATA TFs used to construct the phylogenetic NJ tree is shown in Additional file 1: Table S1.

\section{Motif analysis of A. oryzae and other Aspergillus GATA transcription factors}

MEME was used to predict and analyze motifs of $A$. oryzae GATA proteins, which were visualized using TBtools (Chen et al. 2011). The parameters were set to zero or one of a contributing motif site per sequence, and the numbers of motifs chosen was five; motif widths were set to 6 and 50 (Wu et al. 2016). The other parameters were set to default values. Each motif was individually checked so that only motifs with e-value of $<1 \mathrm{e}-10$ were retained for motif detection in A. oryzae GATA proteins.

\section{Effects of temperature and salinity treatment on $A$. oryzae growth}

A. oryzae 3.042 (CICC 40,092), the main fermentation strain used in industry, was selected to test the growth of $A$. oryzae under temperature and salt stress. A. ory$z a e$ conidia were inoculated in fresh potato dextrose agar (PDA) medium and cultured at $22,25,30,35$ and $42{ }^{\circ} \mathrm{C}$ for $72 \mathrm{~h}$ to investigate the effects of temperature; the optimum growth temperature of $A$. oryzae, $30^{\circ} \mathrm{C}$, was used as the control temperature. PDA media with final salt concentration of 5.0, 10.0, 12.5 and $15.0 \mathrm{~g} / 100 \mathrm{~mL} \mathrm{NaCl}$ were prepared to assess the effects of salinity stress on A. oryzae. Medium without salt was used as the control 
medium. Two microliters of freshly prepared $A$. oryzae suspension containing $1 \times 10^{7}$ conidia were inoculated on the medium to analyze phenotypes. To determine the effect of these two abiotic stresses on fungal viability, $100 \mu \mathrm{L}$ of $1 \times 10^{7}$ conidia suspension was inoculated per plate covered with cellophane (Solarbio, Beijing, China); the fungal mycelia were collected after $72 \mathrm{~h}$ of incubation. The fungal mycelia were then dried overnight, and the dry biomass was tested. Material for RNA extraction was also collected simultaneously. The experiments were performed in triplicate.

\section{Quantitative real-time polymerase chain reaction (qRT-PCR) for analyzing the expression of $A$. oryzae GATA TFs in response to temperature and salinity stress}

Total RNA was extracted using an Omega plant RNA kit (Omega Bio-Tek, Georgia, USA) according to the manufacturer's instructions. One microgram of RNA was reverse-transcribed into cDNA using PrimeScript ${ }^{\mathrm{TM}}$ RT reagent with the gDNA Eraser kit (TaKaRa, Dalian, China). A. oryzae GATA TF primers were designed using the Primer-BLAST tool (https://www.ncbi.nlm.nih.gov/ tools/primer-blast) (Additional file 2: Table S2). Gene expression levels were determined by perfoming qRTPCR on a Bio-rad CFX96 Touch instrument (Bio-Rad, USA) using TB Premix Ex Taq II (TaKaRa) according to the manufacturer's instructions. Data were analyzed using Bio-rad CFX96 software and the $2^{-\Delta \Delta C_{T}}$ method (Livak and Schmittgen 2001). The histone H1 gene was used as the reference gene in qRT-PCR analysis.

\section{Construction of protein-protein interaction network}

Protein-protein interaction (PPI) data were obtained from the online database of STRING (https://string-db. org/), which is an open source software for predicting and visualizing complex networks. These interactions were derived from literature regarding experimental validation of physical interactions and enzymatic reactions associated with signal transduction pathways. The PPI networks were visualized in biological graph-visualization tool Cytoscape with the nodes representing proteins/ genes (Pathan et al. 2015).

\section{Results}

Characteristics of $A$. oryzae GATA TFs

BLASTP analysis was used to check predicted the GATA TFs from the $A$. oryzae 3.042 genome. All potential $A$. oryzae GATA proteins were used to identify ZnF_GATA domains (PF00320) using HMMER3.1. In total, seven $A$. oryzae GATA TFs were identified, and were named AoAreA, AoAreB, AoLreA, AoLreB, AoNsdD, AoSnf5 and AoSreA corresponding to the names of fungal orthologs (Table 1 ). The lengths of the $A$. oryzae GATA TFs ranged from 313 (AoAreB) to 867 (AoAreA) amino acid. The details of these $A$. oryzae GATA TFs, such as ZnF_GATA motif type, number of ZnF_GATA domains, sizes of the deduced peptides, and their homologous gene IDs, are listed in Table 1.

The GATA DNA binding domain is a conserved typeIV zinc-finger motif containing the Cys- $\mathrm{X}_{2}$-Cys- $\mathrm{X}_{17-20^{-}}$ Cys- $\mathrm{X}_{2}$-Cys motif. The zinc-finger motifs of Cys- $\mathrm{X}_{2}$-Cys $-\mathrm{X}_{17-20}$-Cys $-\mathrm{X}_{2}$-Cys differed among the seven $A$. oryzae GATA proteins. Six A. oryzae GATA domains contained the Cys- $\mathrm{X}_{2}$-Cys- $\mathrm{X}_{17 / 18}$-Cys- $\mathrm{X}_{2}$-Cys motif as reported in other fungi, while 20 residues were present in the zincfinger loop of AoSnf5 between the Cys- $\mathrm{X}_{2}$-Cys motifs, which has rarely been found in fungi (Teakle and Gilmartin 1998; Scazzocchio 2000) (Table 1; Fig. 1a). Interestingly, AoSreA harbored two highly conserved type-IV zinc-finger motifs with Cys- $\mathrm{X}_{2}-\mathrm{Cys}-\mathrm{X}_{17}-\mathrm{Cys}-\mathrm{X}_{2}$-Cys (Table 1; Fig. 1a), which usually occur in animals (Patient and Mcghee 2002). Apart from the ZnF_GATA domain, additional domains such as TFIIB zinc-binding domain, AreA-N, SNF5/INI1, and PAS were also characterized (Table 1; Fig. 1b). Previous studies have demonstrated that the PAS domain mainly functions in sensing environmental or physiological signals including oxidative and heat stress (Nan et al. 2011; Corrada et al . 2016). Therefore, extra domains present in A. oryzae GATA may also play the same role in diverse environmental stresses

Table 1 The characteristics of A. oryzae GATA TFs

\begin{tabular}{|c|c|c|c|c|c|c|}
\hline Name & Protein ID & Peptide (aa) & ZnF_GATA Motif type & $\begin{array}{l}\text { Number domain of } \\
\text { ZnF_GATA }\end{array}$ & Homologous ID & Extra domain \\
\hline AoSreA & EIT82081.1 & 567 & Cys- $X_{2}-$ Cys- $X_{17}-$ Cys- $X_{2}$-Cys & 2 & KOC08900.1 & TFIIB zinc-binding \\
\hline AoAreB & EIT79032.1 & 313 & Cys- $X_{2}-$ Cys- $X_{17}-$ Cys- $X_{2}$-Cys & 1 & XP_002379623.1 & TFIIB zinc-binding \\
\hline AoAreA & EIT72728.1 & 867 & Cys- $X_{2}-$ Cys- $X_{17}-$ Cys- $X_{2}$-Cys & 1 & RAQ50831.1 & AreA_N \\
\hline AolreB & EIT79273.1 & 496 & Cys- $X_{2}-$ Cys- $X_{18}-$ Cys- $X_{2}$-Cys & 1 & RAQ50386.1 & PAS \\
\hline AoNsdD & EIT79449.1 & 504 & Cys- $X_{2}-$ Cys- $X_{18}-$ Cys- $X_{2}$-Cys & 1 & KOC07076.1 & - \\
\hline AoLreA & EIT77832.1 & 283 & Cys- $X_{2}-$ Cys- $X_{18}$-Cys- $X_{2}$-Cys & 1 & XP_002384232.1 & PAS \\
\hline AoSnf5 & EIT78280.1 & 570 & Cys- $X_{2}-$ Cys- $X_{20}-$ Cys- $X_{2}$-Cys & 1 & XP_022385751.1 & SNF5/INI1 \\
\hline
\end{tabular}




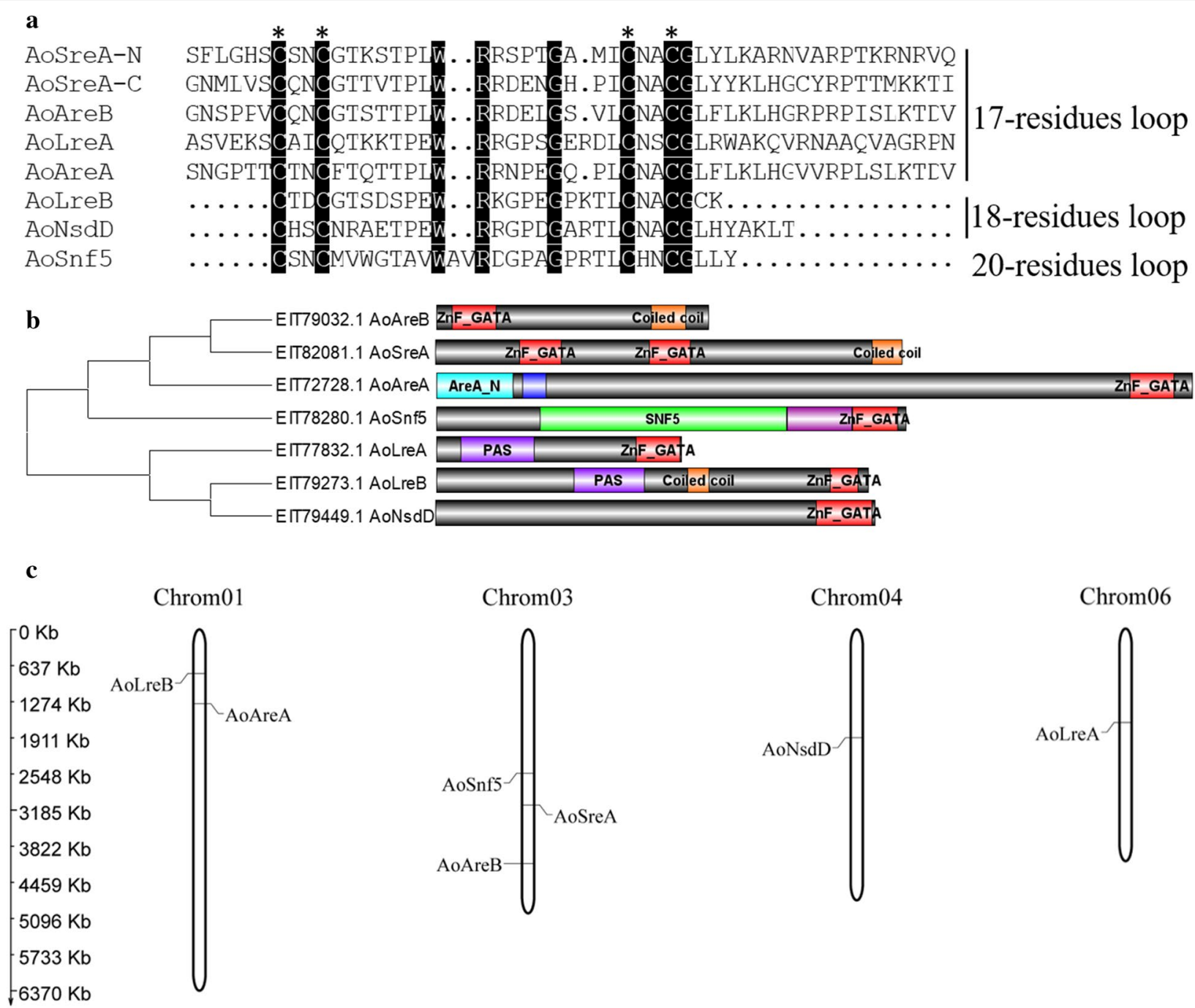

Fig. 1 Alignment of conserved domain, prediction of functional domains, and chromosomal location of A. oryzae GATA TFs. a Alignment of the DNA interacting domain of $A$. oryzae GATA TFs. Cysteines from the Cys- $\mathrm{X}_{2}-\mathrm{Cys}_{\mathrm{s}} \mathrm{X}_{17 / 18 / 20^{-}}$-Cys- $\mathrm{X}_{2}-\mathrm{Cys}$ domain are indicated by an asterisk above the sequence alignment. The 17,18 , and 20 numbers indicate the amino acid residues between $C y s-X_{2}-C y s$. $\mathbf{b}$ Seven $A$. oryzae GATA proteins were aligned and clustered using MEGA6.0, and their ZnF_GATA domains are shown in red beside the neighbor-joining tree. c The distribution of $A$. oryzae GATA TFs on chromosomes. The vertical columns represent chromosomes; gene names are shown at the side of chromosomes

and may facilitate the functional analysis of A. oryzae GATA TFs.

In addition, the chromosomal location of $A$. oryzae GATA TFs revealed their random distribution in the $A$. oryzae genome. Here, the seven GATA TFs of $A$. oryzae 3.042 were mapped to the first complete genome of $A$. oryzae strain RIB40. The chromosomal distribution of A. oryzae GATA TFs was visualized using the MapChart program. The seven $A$. oryzae GATA TFs were randomly distributed on chromosomes 1, 3, 4, and 6 (Fig. 1c). Interestingly, AoAreB, AoSreA, and AoSnf5 clustered into the same subgroup in the NJ tree (Fig. 1b) and were distributed on the same chromosome, which indicates a close evolutionary relationship among them. The chromosomal location of A. oryzae GATA TFs may assist in determining the exact sequence of events.

\section{Phylogenetic analysis of the Aspergillus GATA TFs}

A NJ_tree was constructed using MEGA6.0 for multiple sequence alignment of all Aspergillus GATA TFs with 1000 bootstrap replications to analyze phylogenetic relationships between the $A$. oryzae GATA TFs and other Aspergillus GATA TFs with ZnF_GATA domains. All the Aspergillus GATA TFs were divided into seven subgroups in the $\mathrm{NJ}$ tree based on the number of $\mathrm{ZnF}_{-}$ GATA domains and zinc finger motifs of GATA domain 
sequences with other Aspergillus GATA TFs from FTFD, including six known subgroups of WC1, WC2, NSDD, SRP, ASD4, NIT2 and one unknown function subgroup (Fig. 2). Seven A. oryzae GATA TFs were scattered in the six subgroups with other Aspergillus GATA TFs, functions of which have been reported, while the novel AoSnf5 GATA TF also clustered in the NSDD subgroups together with AoNsdD. The function of the different
GATA subgroups vary. For example, the GATA TFs of WC1 and WC2 subgroups are mainly involved in the regulation of blue- and red-light responses (Purschwitz et al. 2008; Purschwitz et al. 2013). Nitrogen regulation is regulated by the process of nitrogen catabolite repression which controls gene expression via GATA TFs of the NIT2 and ASD4 subgroup in yeasts and filamentous fungi (Pfannmüller et al. 2017; Pomraning et al. 2017;

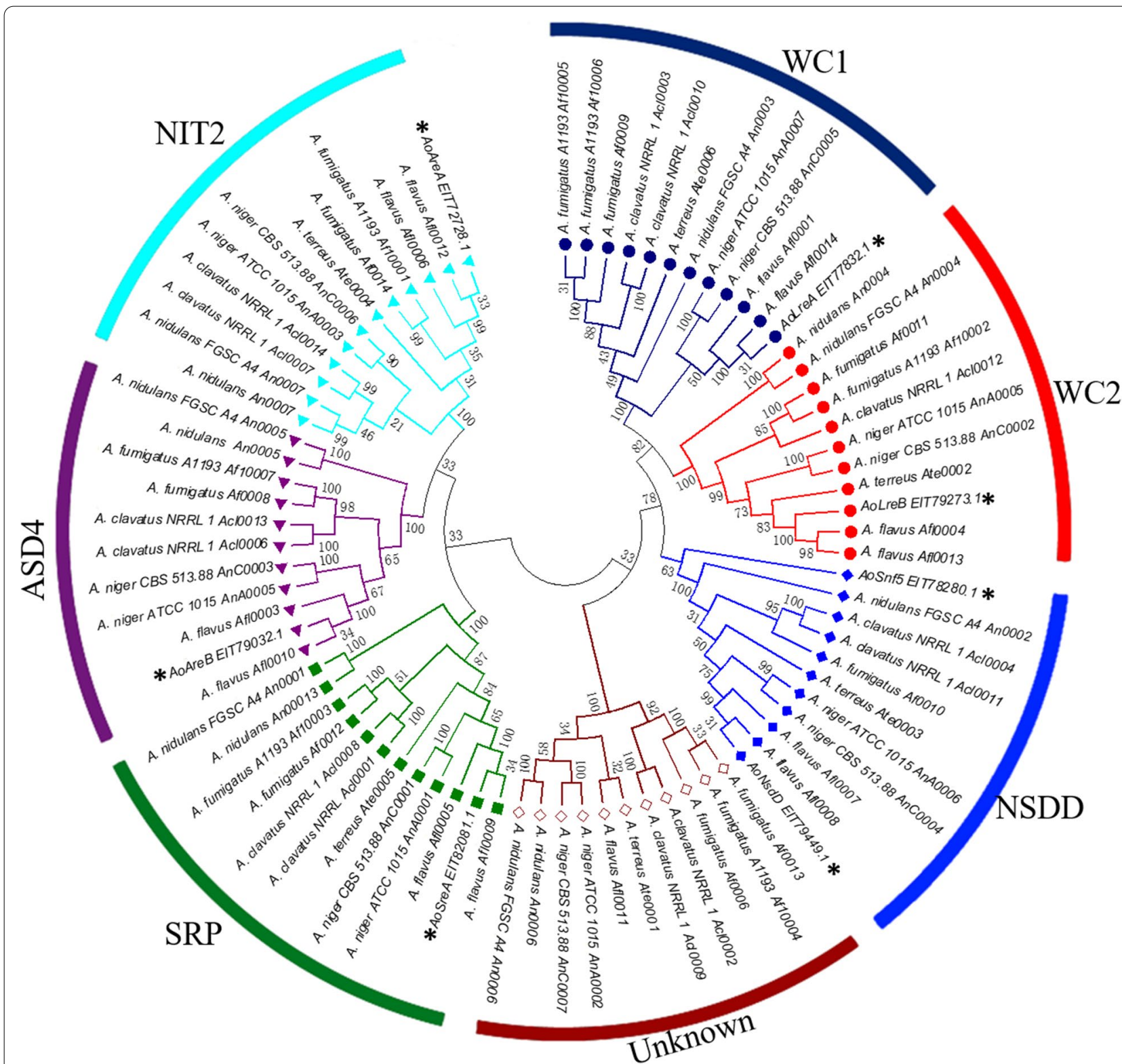

Fig.2 Phylogenetic analysis of A. oryzae and other Aspergillus TFs. GATA protein sequences were aligned using ClustalW in MEGA6.0 software using default parameters. The consensus NJ tree represents 1000 bootstrap replications. Bootstrap values are displayed as nodes. The protein sequences of Aspergillus GATA TFs were downloaded from FTFD. The Aspergillus GATA TFs are classified into seven subgroups in the NJ tree, including one group with unknown function. Seven A. oryzae GATA TFs are scattered in six known subgroups, and the novel AoSnf5 also clustered in the NSDD subgroups together with AoNsdD 
Michielse et al. 2014). Therefore, the AoLreA, AoLreB, AoAreA, and AoAreB divided into WC1, WC2, NIT2, and ASD4 subgroups, respectively, might also be involved in light responses or nitrogen regulation as reported. In addition, $N s d D$ has been shown not only to affect sexual and asexual reproduction, but also secondary metabolism in Aspergillus (Lee et al. 2014, 2016), which could help in determining the function of AoNsdD and Aosnf5 assigned to the NSDD subgroup.

\section{Analysis of conserved motifs in A. oryzae GATA TFs}

To obtain insights regarding the diversity of motif compositions in $A$. oryzae GATA TFs, the conserved motifs in the A. oryzae and other Aspergillus GATA TFs were predicted using the MEME4.11.4 online software. In total, five conserved motifs were identified. The relative locations of these motifs within the protein are shown in Fig. 3. The identified consensus sequence of the five motifs is shown in Additional file 4: Figure S1. A typical zinc-finger structure composed of motif 1 and motif 2 was observed in all Aspergillus GATA TFs; however, the compositions of GATA TF motifs also contained different variable regions. As expected, GATA members that had similar motif compositions could be clustered into one subgroup, which suggested they may perform similar genetic functions within the same subgroups. In addition, the motif distribution further confirmed the accuracy of the phylogenetic relationship of Aspergillus GATA TFs. The distribution of motifs in different subgroups indicated functional differentiation in GATA TFs during evolution.

\section{Effects of different temperature and salinity treatments on the growth of $A$. oryzae}

Temperature and salt concentration are two of the most important environmental factors affecting the growth and fermentation of $A$. oryzae during fermentation (Chen et al. 2011; Bechman et al. 2012; Wang et al. 2013). Therefore, we investigated the growth of $A$. oryzae under different temperature and salt concentration stresses. The optimum temperature for $A$. oryzae growth usually ranges from 30 to $35^{\circ} \mathrm{C}$. Low and high temperatures

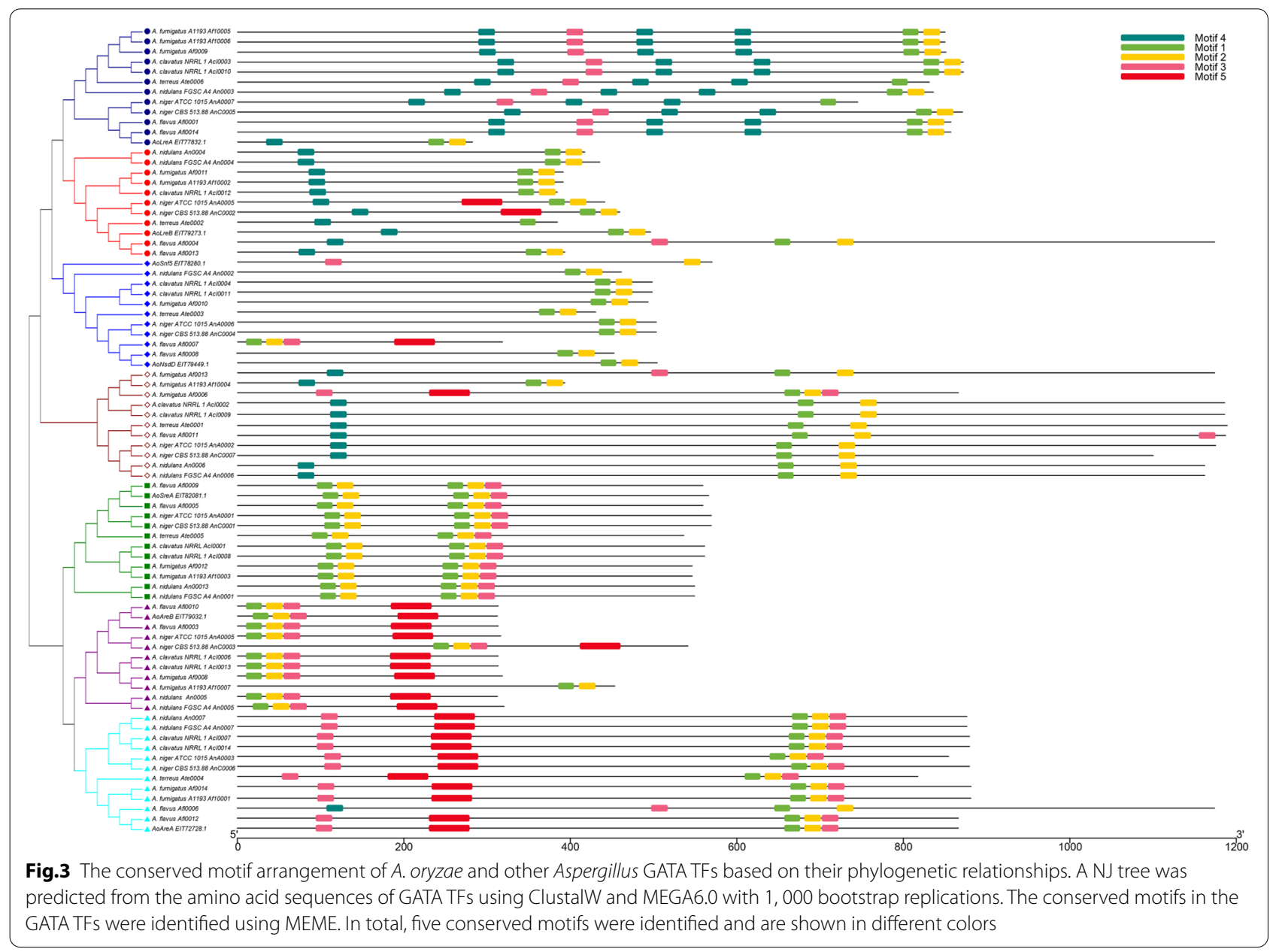


significantly inhibited mycelial growth, especially at the temperature of 22 and $42{ }^{\circ} \mathrm{C}$ (Fig. $4 \mathrm{a}(\mathrm{a}-\mathrm{e})$, b). In addition, high salt concentration also significantly inhibited hyphal growth and differentiation of $A$. oryzae, and the inhibitory effect increased with salt concentration (Fig. $4 \mathrm{a}(\mathrm{f}-\mathrm{j})$, c). Furthermore, the formation and development of $A$. oryzae spores, which shows yellow-green color in the middle of the fungal colony, were also inhibited under low- and high-temperature and high salinity stresses (Fig. 4a, d and e).

\section{Expression patterns of $A$. oryzae GATA TFs in response to temperature and salinity stresses}

To determine the roles of $A$. oryzae GATA TFs in response to abiotic stresses, we analyzed the expression level of the seven $A$. oryzae GATA TFs using qRTPCR in $A$. oryzae that grew at different temperatures and salt concentrations (Fig. 5). and observed that their expression varied under different temperatures and salt stresses. With the exception of the AoSnf5, the six other A. oryzae GATA TFs strongly responded to low or high temperatures (Fig. 5a). AoSreA and AoNsdD showed the same expression trend, as they were significantly induced at low temperature $\left(22{ }^{\circ} \mathrm{C}\right)$ and inhibited at high temperature $\left(42{ }^{\circ} \mathrm{C}\right)$ compared with $\mathrm{CK}\left(30{ }^{\circ} \mathrm{C}\right)$. In addition, AoAreB, AoLreA, and AoLreB, especially AoAreB, were remarkably upregulated at high temperature compared to CK $\left(30^{\circ} \mathrm{C}\right)$ (Fig. 5a). Interestingly, only AoAreA was inhibited at both low and high temperature. Furthermore, AoAreA, AoSreA, and AoAreB were significantly downregulated under high-salt concentration stress, while AoLreA, AoNsdD, and AoSnf5 were upregulated in the presence of 5.0 and $10.0 \mathrm{~g} / 100 \mathrm{~mL} \mathrm{NaCl}$ (Fig. 5b). In addition, we compared the results of qRT-PCR analysis with the transcriptional data of the seven TFs in response to salt stress. We observed strong correlation between the results of qRT-PCR and those obtained using RNA-seq (Pearson correlation, $R^{2}=0.9044$ (Additional file 5: Figure S2). Together, the results highlighted the importance of $A$. oryzae GATA TFs in response to temperature and high salt stresses and provided a basis for future studies into the function of $A$. oryzae GATA in abiotic stresses.

\section{PPI network of $A$. oryzae GATA TFs}

To analyze the functions of $A$. oryzae GATA TFs, a PPI network was constructed using the data from the STRING database, and only two independent PPI network of AoAreA and AoSreA was obtained (Fig. 6a, b). Furthermore, we observed that both AoAreA and AoSreA interacted with $\mathrm{CreA}$, and that $\mathrm{CreA}$ deletion mutants showed less conidiation than the wild type and sensitivity to salt stress (Hou et al. 2018). Therefore, the expression levels of AoAreA, AoSreA, and AoCreA were analyzed under temperature and salt stresses. AoSreA and $\mathrm{AoCreA}$ showed the same expression patterns under both low and high temperature stresses, while AoAreA and $\mathrm{AoCreA}$ exhibited opposite expression level at 22 ${ }^{\circ} \mathrm{C}$ (Fig. 6c). Interestingly, these three genes showed the same expression patterns under high salt concentration stress (Fig. 6d), which demonstrates that $A o C r e A$ may be positively coregulated by both AoAreA and AoSreA under salt stresses. Additionally, glutathione S-transferase (CADAORAP00007152), which is critical to abiotic stress was also found in the network of AoAreA (Favaloro et al. 2000). These results will be beneficial for identifying important proteins and biological modules that interact with $A$. oryzae GATA TFs and understanding the roles of A. oryzae GATA TFs in response to abiotic stresses. The detailed information regarding the proteins in the PPI network is listed in Additional file 3: Table S3.

\section{Discussion}

Transcription factors (TFs) regulate expression of genes that mediate growth processes and environmental response and are employed as a principal source of the diversity and change that underlie evolution (Riechmann and Ratcliffe 2000). Fungal GATA TFs are mainly involved in nitrogen metabolism (Michielse et al. 2014; Pfannmüller et al. 2017), light responses (Purschwitz et al. 2008; Fuller et al. 2013), siderophore biosynthesis and matingtype switching (Jung and Kronstad 2011). Few fungal GATA TFs, such as the SreA, SreB, LreA, LreB, GLN3, and GAT1, also participate in response to abiotic stresses, (Chung et al. 2020; Crespo et al. 2001; Purschwitz et al. 2008; Fuller et al. 2013; Marty et al. 2015). In this study, we focused on the GATA TF family in A. oryzae 3.042 to define the genetic characteristics and improve our understanding regarding their role in response to temperature and salinity stresses. Seven GATA TFs were identified from the A. oryzae 3.042 genome using an HMM model, which was similar to the number of GATA TFs identified in the model fungi, Fusarium graminearum and Botrytis cinerea, which contain 7 and 7 GATA TFs, respectively (Zhang et al. 2014). The number of the GATA TFs is conserved among $A$. clavatus, $A$. flavus, A. fumigatus, $A$. nidulans, and $A$. niger that possess six GATA TFs, suggesting that the composition of GATA TFs in filamentous fungi is identical (Kim et al. 2006; Kobayashi et al. 2007). Interestingly, A. oryzae contains one more GATA TF (AoSnf5) compared to these Aspergillus. Furthermore, we also detected the homologous gene of AoSnf5 in the genomes of Aspergillus species, which was annotated as unnamed or hypothetical protein; however, AoSnf5, encoding a GATA protein with 20 residues between the Cys- $\mathrm{X}_{2}$-Cys motifs, was identified as an Aspergillus GATA TF for the first time. 

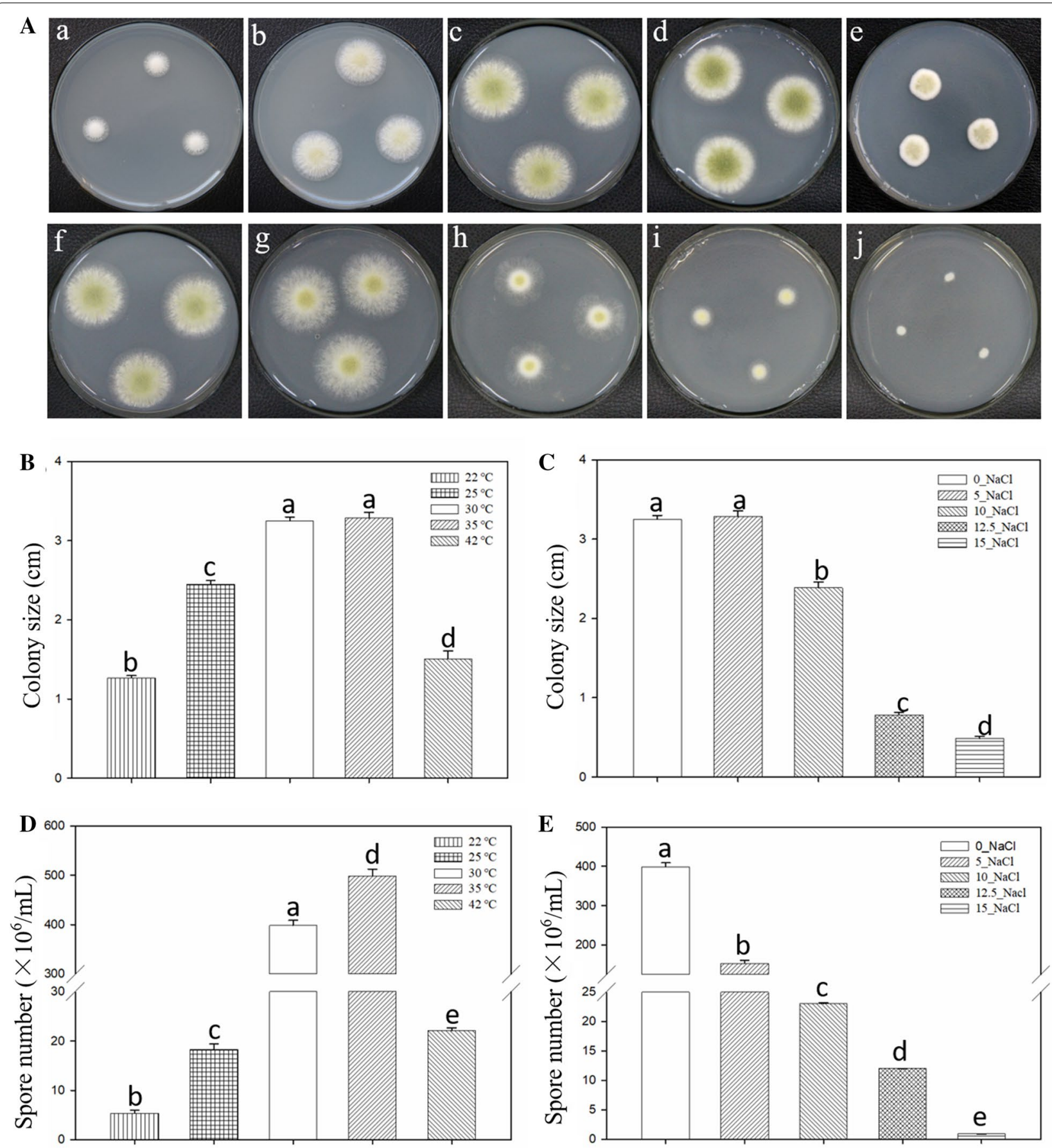

Fig. 4 A. oryzae hyphal growth and differentiation under different stress factors for $72 \mathrm{~h}$. a The phenotypes of $A$. oryzae under temperature and salinity stress. (a-e) Phenotypes of $A$. oryzae exposed to different temperature stresses $\left(22,25,30,35\right.$, and $42{ }^{\circ} \mathrm{C}$ from left to right). (f-j) $0.0,5.0,10.0$, 12.5 , and $15.0 \mathrm{~g} / 100 \mathrm{~mL} \mathrm{NaCl}$ was used for salinity stress. $\mathbf{b}$ and $\mathbf{c}$ Colony diameter was determined by measuring diameter under different stress conditions. $\mathbf{d}$ and $\mathbf{e}$ Spore numbers were counted under temperature and salinity stresses. The spores were collected from colonies and suspended in sterile distilled water to obtain the spore suspension, and then the spore numbers were counted using a hemocytometer. The optimum growth temperature of $\mathrm{A}$. oryzae $\left(30^{\circ} \mathrm{C}\right)$, was used as the control temperature in the experiment. The PDA medium without $\mathrm{NaCl}$ used as the control in the salt treatment experiments. Results represent the average of three repetitions \pm SEM $(n=3)$. Different letters in the bar chart represent significant differences ( $p<0.01$, Duncan's multiple range test); the same letters in the bar chart represent absence of significant difference when compared to the control 

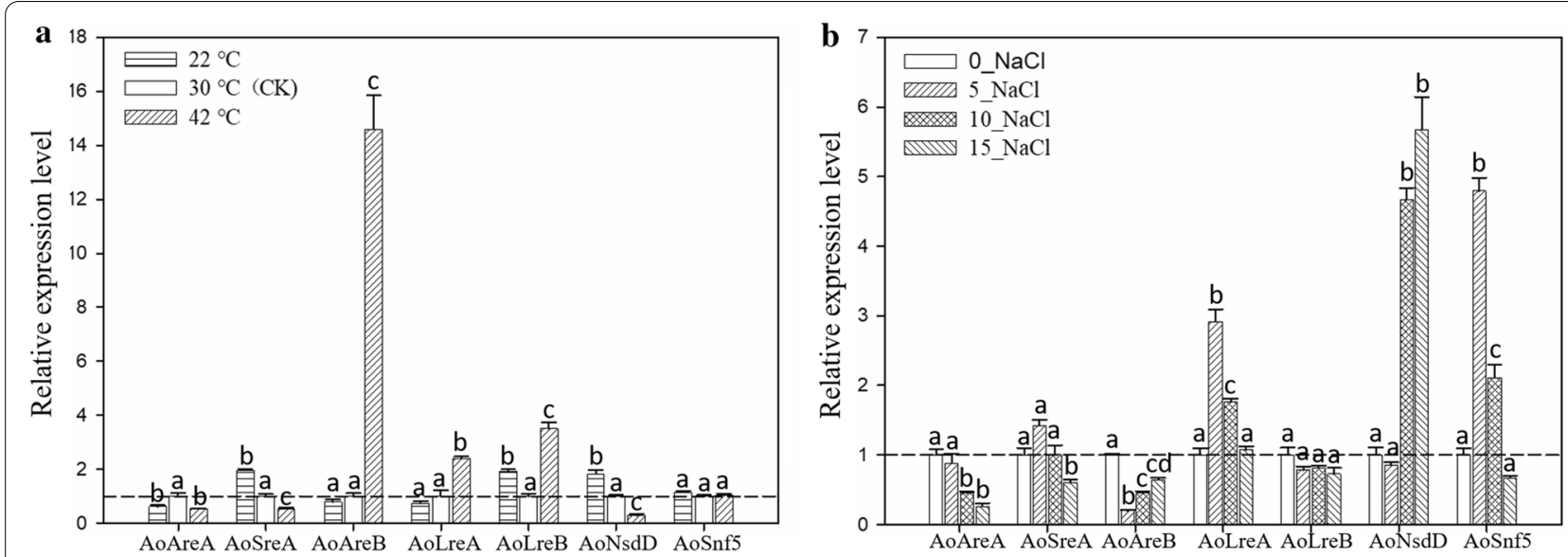

Fig. 5 Expression levels of A. oryzae GATA TFs in response to temperature and salt stresses. a The relative expression levels of A. oryzae GATA TFs responding to low- and high-temperature stresses. $\mathbf{b}$ The expression patterns of $A$. oryzae GATA TFs under different salt concentration stresses. The optimum growth temperature of $A$. oryzae $\left(30^{\circ} \mathrm{C}\right)$ was used as the control temperature $(\mathrm{CK})$ in the experiment. PDA medium without $\mathrm{NaCl}$ was used as the control (CK) under salt stress. Results show the average of three repetitions $\pm S E M(n=3)$. Different letters represent significant differences $(p<0.01$, Duncan's multiple range test); same letters represent lack of significant difference when compared to the control

Although most GATA domains harbor a class-IV zinc-finger motif, their structure varies among kingdoms (Lowry and Atchley 2000). In plants, most GATA domains have a single Cys- $\mathrm{X}_{2}$-Cys- $\mathrm{X}_{18}$-Cys- $\mathrm{X}_{2}$-Cys motif, but some harbor more than two zinc-finger motifs or 20 residues within zinc-finger loops (Reyes et al. 2004; Behringer and Schwechheimer 2015). In animals, the GATA domain harbors two zinc-finger motifs with Cys$\mathrm{X}_{2}$-Cys- $\mathrm{X}_{17}$-Cys- $\mathrm{X}_{2}$-Cys, but only the $\mathrm{C}$-terminal finger is associated with DNA binding (Patient and Mcghee 2002). Fungal GATA TFs are combination of both animal and plant GATA TFs in terms of the amino acid residues present in the zinc-finger loop (Teakle and Gilmartin 1998). The majority of fungal GATA TFs contain a single zinc-finger domain and fall into two different categories: animal-like with 17-residue loops $\left(\mathrm{Cys}-\mathrm{X}_{2}-\right.$ Cys- $\mathrm{X}_{17}-$ Cys- $\mathrm{X}_{2}$-Cys), and plant-like with 18-residue loops (Cys- $\mathrm{X}_{2}$-Cys- $\mathrm{X}_{18}$-Cys- $\mathrm{X}_{2}$-Cys) (Teakle and Gilmartin 1998; Scazzocchio 2000; Patient and Mcghee 2002). Nineteen- and 20-residue zinc-finger loops (Cys- $\mathrm{X}_{2}$-Cys$\mathrm{X}_{19-20}$-Cys- $\mathrm{X}_{2}$-Cys) are also found in fungi, albeit rarely (Scazzocchio 2000; Maxon and Herskowitz 2001). With the exception of the 17- and 18-residue zinc-finger loops in A. oryzae GATA TFs, the novel AoSnf5 contains 20 residues in the zinc-finger loops $\left(\mathrm{Cys}-\mathrm{X}_{2}-\mathrm{Cys}-\mathrm{X}_{20}-\mathrm{Cys}-\mathrm{X}_{2}\right.$ Cys), which are rarely found in fungi (Table1 and Fig. 1). To the best of our knowledge, this is the first study to identify GATA TFs with 20-residue zinc-finger loops in Aspergillus. In addition, AoSreA harbors two ZnF-GATA domains of Cys- $\mathrm{X}_{2}$-Cys- $\mathrm{X}_{17}$-Cys- $\mathrm{X}_{2}$-Cys type, which is the typical GATA characteristic of animals (Lowry and Atchley 2000; Patient and Mcghee 2002). Therefore, the features of $A$. oryzae GATA TFs demonstrated that A. oryzae GATA TFs might be the combination of both plant and animal GATA TFs, which is consistent with the report showing that fungal GATA TFs are combinations of both plant and animal GATA TFs in terms of the numbers of $\mathrm{ZnF}$-GATA domains and amino acid residues present in the zinc-finger loop.

In the phylogenetic tree, seven $A$. oryzae GATA proteins and other Aspergillus GATA TFs were classified into seven subgroups. Consistent with the report of Kobayashi et al. (2007), six known A. oryzae GATA TFs were classified into six functional subgroups based on the number of ZnF_GATA domains and zinc finger motifs of GATA domain sequences with other Aspergillus GATA TFs from FTFD in the phylogenetic NJ tree. In addition, 11 Aspergillus GATA TFs clustered together in an unnamed subgroup (Fig. 2). The phylogenetic positions of fungal GATA TFs are different from those of animal and plant GATA TFs, and most fall into independent clusters. Fungal GATA TFs can be divided into seven phylogenetic subgroups named SRP, NIT2, ASD4, WC1, WC2, NSSDD, and SFH1(Kim et al. 2006; Yu et al. 2019). In this study, all the Aspergillus GATA TFs were divided into seven subgroups consistent with the phylogenetic analysis of fungal GATA TFs, and the unnamed subgroup of Aspergillus GATA TFs might correspond to the SNF1 subgroup in the NJ tree. The GATA TFs of the SFH1 subgroup contain a SNF5/SMARCB1/INI1 domain characteristic of the Swi/Snf family of chromatin remodeling complex in S. cerevisiae (Klochendler-Yeivin and Yaniv 2001). The biological functions of SFH1 GATA factors in filamentous ascomycetes have not yet been characterized. 

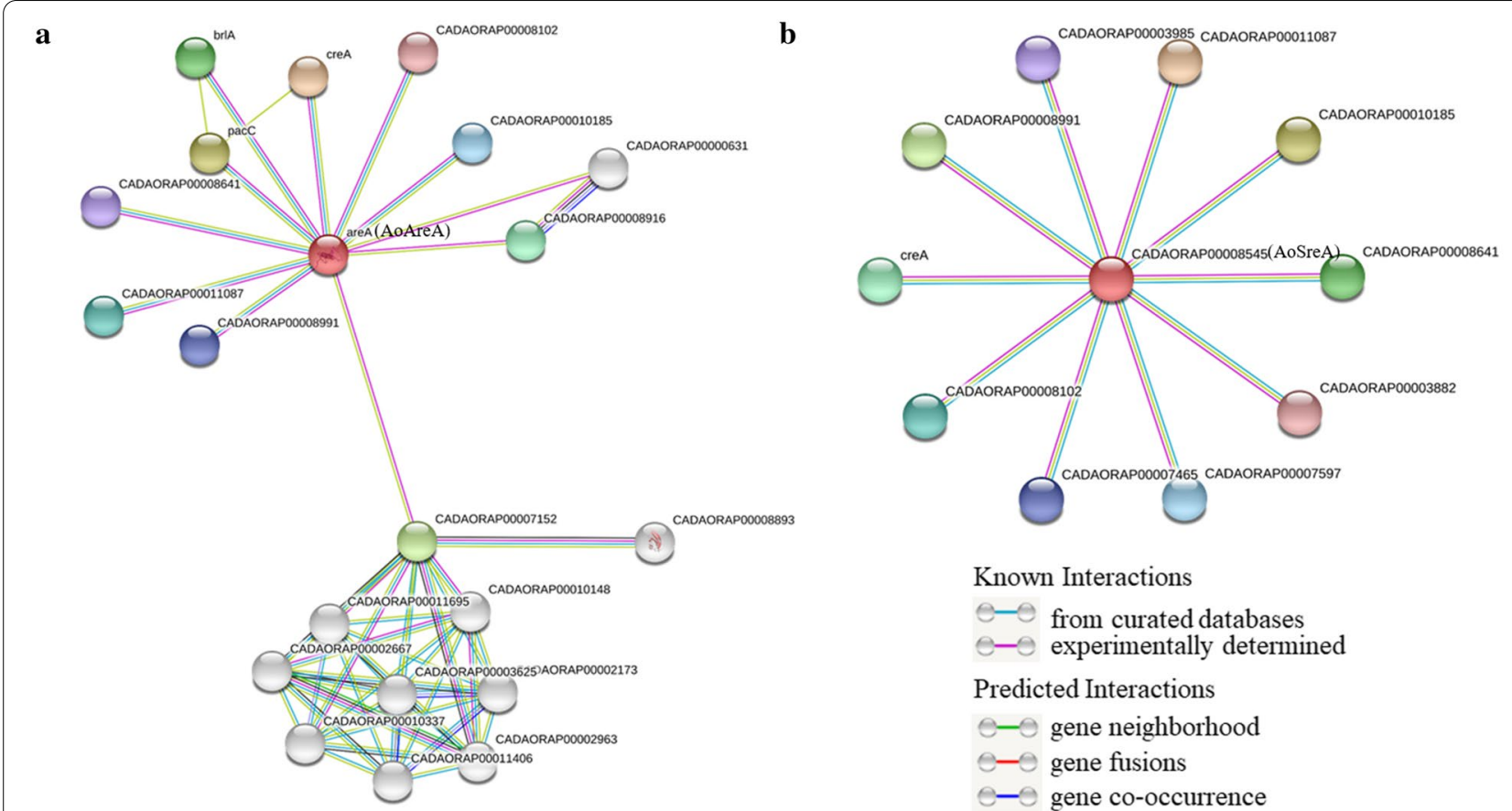

Known Interactions

- - from curated databases

e-९ experimentally determined

Predicted Interactions

¿-९ gene neighborhood

¿-९ gene fusions

e- gene co-occurrence
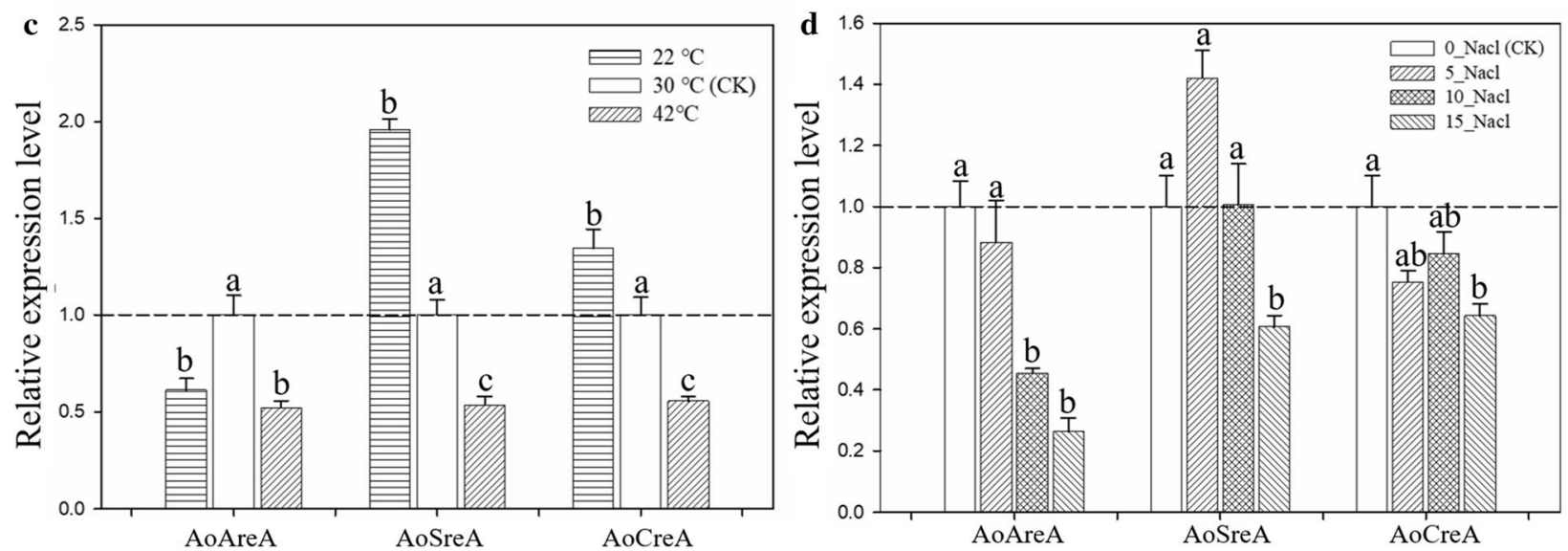

Fig. 6 Protein-protein interaction (PPI) network of A. oryzae GATA TFs. a, b The PPI network of AoAreA and AoSreA. (C, D) The relative expression levels of AoAreA and AoSreA were consistent with that of the interaction partner, AoCreA $(p<0.01, n=3)$. The optimum growth temperature of $A$. oryzae $\left(30^{\circ} \mathrm{C}\right.$ ) was used as the control temperature (CK) in the experiment. PDA medium without NaCl was used as the control (CK) under salt stress. The same letters represent lack of significant difference compared to the control when assessed using Duncan's multiple range test

Interestingly, AoSnf5, a newly identified GATA TF in our study, also contained a SNF5 domain similar to that present in the SFH1 subgroup GATA TFs; however, AoSnf5 clustered in NSDD subgroup together with AoNsdD (Fig. 2). Although we cannot explain this phenomenon yet, the identification of AoSnf5 GATA TF enriches the Aspergillus GATA TF family. Additionally, conserved motifs demonstrated that GATA TF members with similar motif compositions could be clustered into one subgroup (Fig. 3), indicating that they may perform similar genetic functions within the same subgroups. In addition, the motif distribution further confirms the accuracy of the phylogenetic relationship of Aspergillus GATA TFs. The analyses of the phylogenetic tree and conserved motifs demonstrated that the GATA TFs among different Aspergillus were evolutionarily conserved and performed similar function within the same subgroups.

Although the GATA motif and DNA binding specificity were conserved, the rest of the protein was not, thereby leading to the same motif serving different purposes 
in various contexts. Deletion of $A$. nidulans AreB has significant phenotypic effects on the utilization of specific carbon sources, confirming its role in the regulation of carbon metabolism. AreB is shown to regulate the expression of AreA regulatory gene suggesting AreB has a range of indirect regulatory effects (ChudzickaOrmaniec et al. 2019). The functional difference between AreB and AreA mainly because of the difference in the component of conserved motifs (Fig. 3). The deletion of GATA $g z f 1$ did not display a growth defect on any of the nitrogen sources tested in Yarrowia lipolytica, which is homologous to nitrogen starvation response gene $A$. nidulans AreA (Pomraning et al. 2017). Therefore, specific motif and structures contained in A. oryzae might perform functions related to special biological processes.

TFs are the key transcriptional regulators that exhibit different expression profiles under distinct physiological and environmental conditions and synchronize stimuli and response. Many studies have revealed the GATA TFs are involved in the regulation of various abiotic stress responses in plants (Peng et al. 2015; Gupta et al. 2017; Nutan et al. 2019) and few fungi (Crespo et al. 2001; Fuller et al. 2013; Marty et al. 2015; Chung et al. 2020). Temperature and salt concentration are two of the most important environmental factors affecting the growth of A. oryzae during fermentation (Machida et al. 2018; Chen et al. 2011; Bechman et al. 2012; Wang et al. 2013). AreA and $A r e B$ function as positive and negative transcriptional regulators that regulat nitrogen and carbon metabolism in Fusarium fujikuroi and A. nidulans (Michielse et al.2014; Pfannmüller et al. 2017; Chudzicka-Ormaniec et al. 2019). The expression level of AoAreA and AoAreB showed opposite trends at high temperature $\left(42{ }^{\circ} \mathrm{C}\right) \mathrm{com}$ pared to that of CK $\left(30{ }^{\circ} \mathrm{C}\right)$ in A. oryzae (Fig. 5a), which indicated AoAreA and AoAreB might also act as negative and positive transcriptional regulators under high-temperature stress, respectively. The clustering of AoNsdD and AoSnf5 in the NSDD subgroup in the NJ tree (Fig. 2) was strongly induced under high salt stress. $N s d D$ has been reported to be a key repressor affecting the quantity of asexual spores in Aspergillus (Lee et al. 2014, 2016), although studies regarding the response of $N s d D$ to adversity stress in Aspergillus are lacking. In addition to the regulation of siderophore biosynthesis and iron metabolism, SreA is also related to the maintenance of cell wall integrity and negatively impacts resistance, as $\triangle$ sre $A$ increases resistance to $\mathrm{H}_{2} \mathrm{O}_{2}$, calcofluor white, and Congo red (Chung et al. 2020). AoSreA was significantly downregulated at $42{ }^{\circ} \mathrm{C}$ and under high salt stress, which indicates that AoSreA might negatively impact high temperature and high salt resistance. In contrast, AoS$r e A$ was significantly upregulated at $22{ }^{\circ} \mathrm{C}$, and a report shows that the $\mathrm{SreB}$ strongly expresses and contributes to filamentous growth at $22{ }^{\circ} \mathrm{C}$ via lipid metabolism in Blastomyces dermatitidis (Marty et al. 2015). The ZnF_GATA domain is conserved in AoSreA and SreB (Additional file 6: Figure S3), which demonstrates that overexpression AoSreA in A. oryzae might also enhance the growth of mycelium at $22{ }^{\circ} \mathrm{C}$. Furthermore, $\mathrm{AoCreA}$, protein of which interacts with AoSreA in the PPI network, has the same expression pattern as $A o S r e A$, indicating that $A o S$ $r e A$ might positively regulate $\mathrm{AoCreA}$ under temperature and high salt stresses. Interestingly, $\mathrm{AoCreA}$ expression was suppressed under high salt stress in A. oryzae, which is in contrast to the results of a previous study showing that $\triangle$ creA mutants of Fusarium graminearum are sensitive to salt stress (Hou and Wang 2018). However, the results provide insights regarding the critical role of SreA in resistance to different temperatures and high salt stresses in A. oryzae.

LreA and $L r e B$, the GATA TFs of WC1 and WC2 subgroups, are involved in the regulation of blue- and redlight responses (Purschwitz et al. 2008; Fuller et al.2013). AoLreA and AoLreB, belonging to WC1 and WC2 subgroups in the NJ tree (Fig. 2), act as a dimer and contain typical PAS dimerization domains shown in Table 1 and Fig. 1b. Previous studies have demonstrated that the PAS domain also functions in sensing environmental or physiological signals including oxidative and heat stress (Nan et al. 2011; Corrada et al. 2016). Therefore, except for the regulation of blue- and red-light responses, the PAS domains present in AoLreA and AoLreB may facilitate the environmental response of $A$. oryzae GATA TFs. Additionally, $L r e A$ and $L r e B$ is a regulatory complex of the global regulator $V e A$, which plays a critical role in environmental stress response in A. cristatus; indeed, the $\Delta v e A$ mutants are more sensitive to high salt, osmotic pressure, and temperature stress (Calvo 2008; Tan et al. 2018). In our study, AoLreA and AoLreB expression increased under high-temperature $\left(42{ }^{\circ} \mathrm{C}\right)$ stress, and AoLreA expression was significantly induced in the presence of 5.0 and $10.0 \mathrm{~g} / 100 \mathrm{~mL} \mathrm{NaCl}$. These results demonstrated that AoLreA and AoLreB might act as a regulatory complex of the global regulator $V e A$ in response to temperature and high salt stresses in A.oryzae.

In summary, we identified and functionally characterized seven GATA TFs proteins from A. oryzae 3.042 genome. Compared to the previous reports, $A$. oryzae contains one more GATA TF (AoSnf5), which encodes a GATA protein with 20 residues between the Cys- $\mathrm{X}_{2}$ Cys motifs. To the best of our knowledge, this is the first study to identify GATA TFs with 20-residue zincfinger loops in Aspergillus. Our results may be useful for elucidating the evolutionary relationships, expression patterns, and functional divergence of GATA TFs in A. oryzae and enrich the Aspergillus GATA TF 
family. In addition, the expression patterns of these $A$. oryzae GATA TFs under distinct environmental conditions provided useful information for further analysis of the function of $A$. oryzae GATA TFs in regulation of various abiotic stress responses in Aspergillus.

\section{Abbreviations}

TF: Transcription factors; NJ tree: Neighbor-joining phylogenetic tree; PPI: Protein-protein interaction network; PDA: Potato dextrose agar.

\section{Supplementary Information}

The online version contains supplementary material available at https://doi. org/10.1186/s13568-021-01212-w.

Additional file 1: Table S1. Sequence IDs of GATA TFs used to construct the neighbor-joining phylogenetic tree.

Additional file 2: Table S2. Primers used in qRT-PCR of A. oryzae GATA gene in response to abiotic stress.

Additional file 3: Table S3. Detailed information regarding the proteins in the PPI network.

Additional file 4: Figure S1. The five structural motifs in A. oryzae GATA TF proteins.

Additional file 5: Figure S2. The expression correlation of GATA TFs between the qRT-PCR results and those obtained using RNA-seq.

Additional file 6: Figure S3. Alignment of the predicted amino acid sequence of AoSreA with SreB. AoSreA and SreB contained several conserved domains including two ZnF_GATA (N-terminal and C-terminal) separated by a cysteine-rich region (CRR) and a conserved C-terminus (CCT) with a predicted coiled-coil domain.

\section{Acknowledgements}

Not applicable.

\section{Authors' contributions}

This work was completed with the efforts of all authors. JC was responsible for data analysis, experiment, and manuscript writing. LG and HB analyzed the Pfam of A. oryzae genes. GJ was responsible for experiments. ZZ provides the chromosomal location methods. $\mathrm{HZ}$ provided the methods of phenotypic analysis. ZB was responsible for the A. oryzae materials and research funding. All the authors have read and approved the final manuscript.

\section{Funding}

This work was supported by the National Natural Science Foundation of China (31900063) to Chunmiao Jiang, the Science and Technology Research Project of Jiangxi Provincial Department of Education (Grant numbers GJJ180630) to Chunmiao Jiang and the Innovation Fund Designated for Graduate Students of Jiangxi Science and Technology Normal University (YC2020-X20) to Gongbo LV.

\section{Availability of data and materials}

The genome-wide transcriptome data of A. oryzae in different growth stages and salt stress treatment have been submitted to NCBI SRA databases under Bioproject Accession PRJNA407002 and PRJNA383095.

\section{Declarations}

Ethics approval and consent to participate

Not applicable.

\section{Consent for publication}

Not applicable.

\section{Competing interests}

The authors declare that they have no conflict of interests.

Received: 25 October 2020 Accepted: 24 March 2021

Published online: 19 April 2021

\section{References}

Bechman A, Phillips RD, Chen J (2012) Changes in selected physical property and enzyme activity of rice and barley koji during fermentation and storage. J Food Sci 77(4-6):M318-M322. https://doi.org/10.1111/j.1750-3841. 2012.02691.x

Behringer C, Schwechheimer C (2015) $\beta$-GATA transcription factors-insights into their structure, regulation, and role in plant development. Front Plant Sci 6:1-12. https://doi.org/10.3389/fpls.2015.00090

Calvo AM (2008) The veA regulatory system and its role in morphological and chemical development in fungi. Fungal Genet Biol 45(7):1053-1061. https://doi.org/10.1016/j.fgb.2008.03.014

Chen T, Xiong S, Jiang S, Wang M, Wu Q, Wei H (2011) Molecular identification of microbial community in Chinese douchi during post-fermentation process. Food Sci Biotechnol 20(6):1633-1638. https://doi.org/10.1007/ s10068-011-0225-0

Chudzicka-Ormaniec P, Macios M, Koper M, Weedall GD, Caddick MX, Weglenski P, Dzikowska A (2019) The role of the GATA transcription factor AreB in regulation of nitrogen and carbon metabolism in Aspergillus nidulans. FEMS Microbiol Lett. https://doi.org/10.1093/femsle/fnz066

Corrada D, Soshilov AA, Denison MS, Bonati L (2016) Deciphering dimerization modes of PAS Domains: computational and experimental analyses of the AhR: ARNT complex reveal new insights into the mechanisms of AhR transformation. PLoS Comput Biol 12:e100498. https://doi.org/10.1371/ journal.pcbi. 1004981

Crespo JL, Daicho K, Ushimaru T, Hall MN (2001) The GATA transcription factors GLN3 and GAT1 link TOR to salt stress in Saccharomyces cerevisiae. J Biol Chem 276:34441-34444. https://doi.org/10.1021/la000749n

Favaloro B, Tamburro A, Trofino MA, Boogna L, Rotilio D, Heipieper HJ (2000) Modulation of the glutathione S-transferase in Ochrobactrum anthropi: function of xenobiotic substrates and other forms of stress. Biochem J 346(2):553-559. https://doi.org/10.1042/0264-6021:3460553

Fernandes CM, Goldman GH, Del Poeta M (2018) Biological roles played by sphingolipids in dimorphic and filamentous fungi. MBio 9(3):e00642-e718. https://doi.org/10.1128/mBio.00642-18

Fuller KK, Ringelberg CS, Loros JJ, Dunlap JC (2013) The fungal pathogen Aspergillus fumigatus regulates growth metabolism, and stress resistance in response to light. MBio 4(2):e00142-e212. https://doi.org/10.1128/ mBio.00142-13

Gupta P, Nutan KK, Singla-Pareek SL, Pareek A (2017) Abiotic stresses cause differential regulation of alternative splice forms of GATA transcription factor in rice. Front Plant Sci 8:1944. https://doi.org/10.3389/fpls.2017.01944

Nan H, Whitelaw ML, Shearwin KE, Dodd IB, Chapman-Smith A (2011) Identification of residues in the $\mathrm{N}$-terminal PAS domains important for dimerization of Arnt and AhR. Nucleic Acids Res 39:3695-3709. https://doi.org/10. 1093/nar/gkq1336

Hou R, Wang C (2018) The function of the carbon metabolism regulator FgCreA in Fusarium graminearum. Sci Agric Sinica 51:257-267. https://doi. org/10.3864/j.issn.0578-1752.2018.02.006

Jung WH, Kronstad JW (2011) The iron-responsive, GATA-type transcription factor cir1 influences mating in Cryptococcus neoformans. Mol Cells 31(1):73-77. https://doi.org/10.1007/s10059-011-0011-0

Kim H, Kim S, Kong S, Park J, Lee YH (2006) A comparative genome-wide analysis of GATA transcription factors in fungi. Genom Inf 4(4):147-160

Kitamoto K (2015) Cell biology of the Koji mold Aspergillus oryzae. Biosci Biotechnol Biochem 79:863-869. https://doi.org/10.1080/09168451.2015. 1023249

Kobayashi T, Abe K, Asai K, Gomi K, Juvvadi PR (2007) Genomics of Aspergillus oryzae. Biosci Biotechnol Biochem 71(3):646-670. https://doi.org/10. $1271 /$ bbb.60550

Chung K, Wu P, Chen Y, Yago Jl (2020) The siderophore repressor SreA maintains growth, hydrogen peroxide resistance, and cell wall integrity in the phytopathogenic fungus Alternaria alternata. Fungal Genet Biol. https:// doi.org/10.1016/j.fgb.2020.103384 
Lee MK, Kwon NJ, Choi JM, Lee IS, Jung S, Yu JH (2014) NsdD is a key repressor of asexual development in Aspergillus nidulans. Genetics 197(1):159-173. https://doi.org/10.1534/genetics.114.161430

Lee MK, Kwon NJ, Lee IS, Jung S, Kim SC, Yu JH (2016) Negative regulation and developmental competence in Aspergillus. Sci Rep 6:28874. https://doi. org/10.1038/srep28874

Livak KJ, Schmittgen TD (2001) Analysis of relative gene expression data using real-time quantitative PCR and the $2^{-\triangle \Delta C T}$ method. Methods 25:402-408. https://doi.org/10.1038/srep28874

Lowry JA, Atchley WR (2000) Molecular evolution of the GATA family of transcription factors: conservation within the DNA-binding domain. J Mol Evol 50:103-115. https://doi.org/10.1109/MWC.2008.4454707

Machida M, Yamada O, Gomi K (2018) Genomics of Aspergillus oryzae: learning from the history of Koji mold and exploration of its future. DNA Res 15:173-183. https://doi.org/10.1093/dnares/dsn020

Marty AJ, Broman AT, Zarnowski R, Dwyer TG, Bond LM, Lounes-Hadj Sahraoui A, Fontaine J, Ntambi JM, Keles S, Kendziorski C, Gauthier GM (2015) Fungal morphology, iron homeostasis, and lipid metabolism regulated by a GATA transcription factor in Blastomyces dermatitidis. PLoS Pathog 11:e1004959. https://doi.org/10.1371/journal.ppat.1004959

Maxon ME, Herskowitz I (2001) Ash1p is a site-specific DNA-binding protein that actively represses transcription. Proc Natl Acad Sci USA 98:1495-1500

Michielse CB, Pfannmuller A, Macios M, Rengers P, Dzikowska A, Tudzynski B (2014) The interplay between the GATA transcription factors AreA, the global nitrogen regulator and AreB in Fusarium fujikuroi. Mol Microbiol 91(3):472-493. https://doi.org/10.1111/mmi.12472

Niehaus EM, Schumacher J, Burkhardt I, Rabe P, Spitzer E, Münsterkötter M, Güldener U, Sieber CMK, Dickschat JS, Tudzynski B (2017) The GATAType transcription factor $\mathrm{Csm} 1$ regulates conidiation and secondary metabolism in Fusarium fujikuroi. Front Microbiol 8:01175. https://doi.org/ 10.3389/fmicb.2017.01175

Nutan KK, Singla-Pareek SL, Pareek A (2019) The Saltol QTL-localized transcription factor OsGATA8 plays an important role in stress tolerance and seed development in Arabidopsis and rice. J Exp Bot 71 (2):684-698. https:// doi.org/10.1093/jxb/erz368

Oberegger H, Schoeser M, Zadra I, Abt B, Haas H (2010) SREA is involved in regulation of siderophore biosynthesis, utilization and uptake in Aspergillus nidulans. Mol Microbiol 41(5):1077-1089. https://doi.org/10.1046/j. 1365-2958.2001.02586.x

Pathan M, Keerthikumar S, Ang CS, Gangoda L, Quek C, Williamson NA, Mouradov D, Sieber OM, Simpson RJ, Salim A, Bacic A, Hill AF, Stroud DA, Ryan MT, Agbinya JI, Mariadason JM, Burgess AW, Mathivanan S (2015) FunRich: an open access standalone functional enrichment and interaction network analysis tool. Proteomics 15(15):2597-2601. https://doi.org/ 10.1002/pmic.201400515

Patient RK, Mcghee JD (2002) The GATA family (Vertebrates and Invertebrates). Curr Opin Genet Dev 12(4):416-422. https://doi.org/10.1016/S0959437X(02)00319-2

Peng X, Wu Q, Teng L, Tang F, Pi Z, Shen S (2015) Transcriptional regulation of the paper mulberry under cold stress as revealed by a comprehensive analysis of transcription factors. BMC Plant Biol 15:108. https://doi.org/10. 1186/s12870-015-0489-2

Pfannmüller A, Leufken J, Studt L, Michielse CB, Sieber CMK, Güldener U, Hawat S, Hippler M, Fufezan C, Tudzynski B (2017) Comparative transcriptome and proteome analysis reveal a global impact of the nitrogen regulators AreA and AreB on secondary metabolism in Fusarium fujikuroi. PLoS ONE 12:e0176194. https://doi.org/10.1371/journal.pone.0176194

Pomraning KR, Bredeweg EL, Baker SE (2017) Regulation of nitrogen metabolism by GATA zinc finger transcription factors in Yarrowia lipolytica. mSphere 2(1):e00038-e117. https://doi.org/10.1128/mSphere.00038-17

Purschwitz J, Müller S, Kastner C, Schöser M, Haas H, Espeso EA, Atoui A, Calvo AM, Fischer R (2008) Functional and physical interaction of blue- and redlight sensors in Aspergillus nidulans. Curr Biol 18(4):255-259. https://doi. org/10.1016/j.cub.2008.01.061

Reyes JC, Muro-Pastor MI, Florencio F (2004) The GATA family of transcription factors in Arabidopsis and rice. Plant Physiol 134(4):1718-1732. https:// doi.org/10.1104/pp.103.037788

Riechmann JL, Ratcliffe OJ (2000) A genomic perspective on plant transcription factors. Curr Opin Plant Biol 3:423-434. https://doi.org/10.1016/ S1369-5266(00)00107-2

Scazzocchio C (2000) The fungal GATA factors. Curr Opin Microbiol 3(2):126131. https://doi.org/10.1016/\$1369-5274(00)00063-1

Schrettl M, Kim HS, Eisendle M, Kragl C, Nierman WC, Heinekamp T, Werner ER, Jacobsen I, IIImer P, Yi H, Brakhage AA, Haas H (2008) SreA-mediated iron regulation in Aspergillus fumigatus. Mol Microbiol 70(1):27-43. https://doi. org/10.1111/j.1365-2958.2008.06376.x

Tan Y, Wang H, Wang Y, Ge Y, Ren X, Ren C, Wang Y, Ren X, Liu Y, Liu Z (2018) The role of the, veA, gene in adjusting developmental balance and environmental stress response in Aspergillus cristatus. Fungal Biol 122(10):952964. https://doi.org/10.1016/j.funbio.2018.05.010

Teakle GR, Gilmartin PM (1998) Two forms of type IV zinc-finger motif and their kingdom-specific distribution between the flora, fauna and fungi. Trends Biochem Sci 23:100-102. https://doi.org/10.1016/S0968-0004(98)01174-8

Voorrips RE (2002) MapChart: software for the graphical presentation of linkage maps and QTLs. J Hered 93(1):77-78. https://doi.org/10.1093/jhered/ 93.1.77

Wang D, Zheng ZY, Feng J, Zhan XB, Zhang LM, Wu JR, Lin CC (2013) A high salt tolerant neutral protease from Aspergillus Oryzae: Purification, characterization and kinetic properties. Appl Biochem Microbiol 49:378-385. https://doi.org/10.1134/S0003683813040170

Wu Z, Cheng J, Cui J, Xu X, Liang G, Luo X, Chen X, Tang X, Hu K, Cheng Q (2016) Genome-wide identification and expression profile of Dof transcription factor gene family in Pepper (Capsicum annuum L.). Front Plant Sci 7(164):574. https://doi.org/10.3389/fpls.2016.00574

Yu M, Yu J, Yong CH, Liu Y (2019) Genome-wide identification and analysis of the GATA transcription factor gene family in Ustilaginoidea virens. Genome. https://doi.org/10.1139/gen-2018-0190

Zhang Y, Zhang K, Fang A, Han Y, Yang J, Xue M et al (2014) Specific adaptation ofUstilaginoidea virensin occupying host florets revealed by comparative and functional genomics. Nat Commun 5:3849. https://doi.org/10.1038/ ncomms4849

\section{Publisher's Note}

Springer Nature remains neutral with regard to jurisdictional claims in published maps and institutional affiliations.

\section{Submit your manuscript to a SpringerOpen ${ }^{\circ}$ journal and benefit from:}

- Convenient online submission

- Rigorous peer review

- Open access: articles freely available online

- High visibility within the field

- Retaining the copyright to your article

Submit your next manuscript at $\boldsymbol{\Delta}$ springeropen.com 